\title{
Spectral transition parameters for a class of Jacobi matrices
}

\author{
by \\ Joanne Dombrowski and Steen Pedersen (Dayton, OH)
}

\begin{abstract}
This paper initially considers a class of unbounded Jacobi matrices defined by an increasing sequence of repeated weights. Spectral parameters are then introduced in various ways to allow the authors to study the nature and location of the spectrum as a function of these parameters.
\end{abstract}

1. Introduction. This paper considers infinite tridiagonal matrices determined by two sequences $\left\{a_{n}\right\},\left\{b_{n}\right\}, a_{n}>0, b_{n}$ real, of the following form:

$$
\begin{aligned}
C & =\left[\begin{array}{ccccc}
b_{1} & a_{1} & 0 & 0 & \ldots \\
a_{1} & b_{2} & a_{2} & 0 & \ldots \\
0 & a_{2} & b_{3} & a_{3} & \ldots \\
\cdot & 0 & a_{3} & b_{4} & \ldots \\
\cdot & \cdot & \cdot & \cdot & \ldots
\end{array}\right], \\
D_{C} & =\left\{x=\left\{x_{n}\right\} \in \ell^{2}: C x \in \ell^{2}\right\} .
\end{aligned}
$$

Additional conditions must be imposed on the sequences $\left\{a_{n}\right\},\left\{b_{n}\right\}$ to claim that this linear transformation is self-adjoint. It is sufficient, for example, to assume the Carleman condition $\sum_{n=1}^{\infty} 1 / a_{n}=\infty$.

If $C$ is self-adjoint, then the spectral theorem asserts that it is unitarily equivalent to a multiplication operator $M_{x}: L^{2}(\mu) \rightarrow L^{2}(\mu)$ defined by $M_{x}: f(x) \mapsto x f(x)$. Furthermore, if $C=\int \lambda d E_{\lambda}$, then the Borel measure $\mu$ is defined by $\mu(\beta)=\left\|E(\beta) \phi_{1}\right\|^{2}$, where $\phi_{1}$ is the first standard basis vector. Note that this vector is a cyclic vector since the subdiagonal entries of the matrix are strictly positive. Since every cyclic self-adjoint Hilbert space operator has a tridiagonal matrix representation of type (1.1) with respect to the basis generated by the cyclic vector, such operators provide canonical models for the study of spectral behavior. It is the aim of this paper to impose conditions on the defining sequences $\left\{a_{n}\right\},\left\{b_{n}\right\}$ that will

2000 Mathematics Subject Classification: 47B36, 47B15, 47B39. 
lead to interesting spectral phenomena. In the following, $\sigma(C)$ will denote the spectrum of the operator defined in (1.1).

The given sequences $\left\{a_{n}\right\},\left\{b_{n}\right\}$ uniquely determine a set of polynomials $\left\{P_{n}(x)\right\}$ defined as follows:

$$
\begin{aligned}
& P_{1}(x)=1, \quad P_{2}(x)=x / a_{1}, \\
& P_{n+1}(x)=\left[\left(x-b_{n}\right) P_{n}(x)-a_{n-1} P_{n-1}(x)\right] / a_{n}, \quad n \geq 2 .
\end{aligned}
$$

When (1.1) defines a self-adjoint operator, these polynomials form an orthonormal basis for the corresponding Hilbert space $L^{2}(\mu)$. They will play a significant role in the proofs of some of the results of this paper.

The key results of this paper may be summarized as follows:

Result 1. Assume $a_{2 n-1}=a_{2 n}=n^{\alpha}, 0<\alpha<\infty, b_{n}=0$, and let $C_{\alpha}$ be the operator determined by (1.1). If $\alpha \leq 1$, then $C_{\alpha}$ has no eigenvalues, and if $\alpha>1$ then $C_{\alpha}$ has pure point spectrum with no finite points of accumulation. Furthermore, if $0<\alpha<1$, then $\sigma\left(C_{\alpha}\right)=\mathbb{R}$, and if $\alpha=1$, then $\sigma\left(C_{\alpha}\right) \cap(-1 / 2,1 / 2)=\emptyset$.

Result 2. Assume $a_{2 n-1}=n+c, a_{2 n}=n+d$, and let $C$ be the operator determined by (1.1). If $c=0$ and $0<d<1 / 2$ then the spectral measure $\mu$ is absolutely continuous and $(-1 / 2+d, 1 / 2-d)$ is a gap in the spectrum. If $c=-d$ and $0<d<1 / 8$ then $\mu$ is absolutely continuous and $(-\sqrt{1 / 4-2 d}, \sqrt{1 / 4-2 d})$ is a gap in the spectrum.

Result 3. Assume $a_{2 n-1}=n+c, a_{2 n}=n+d$, and let $C$ be the operator determined by (1.1). If $-1<c, d+c>0,0 \leq d-c \leq 1$ and $\alpha=(d-c)^{2}-(d-c)+1 / 4$, then for $\alpha>0,(-\sqrt{\alpha}, \sqrt{\alpha})$ is a gap in the essential spectrum of $C$. There are no non-zero eigenvalues in this gap, and the spectral measure $\mu$ is absolutely continuous on the complement of this interval.

These results are closely related to the following theorem, established in [DP02], which will serve as the starting point for the development of the ideas in this paper:

THEOREM 1.1. If $a_{2 n}=a_{2 n-1}=n, n \geq 1, b_{n}=0, n \geq 1$, then (1.1) defines a self-adjoint operator whose corresponding spectral measure is absolutely continuous.

The proof of Theorem 1.1 presented in [DP02] did not identify the spectrum of the operator. It was subsequently observed by Moszyński that there is a gap in the spectrum. More specifically, Moszyński used properties of the Cesàro operator to show that the interval $(-1 / 2,1 / 2)$ is not in the spectrum (so that the spectral measure is identically zero in this interval). This fact can also be established via the argument presented below. The following lemma is needed for this and subsequent arguments. 
Lemma 1.2. If $\left\{x_{n}\right\} \in \ell^{2}$, then the following inequalities hold:

$\sum_{n=1}^{\infty} n^{2}\left|x_{n}-x_{n+1}\right|^{2} \geq \frac{1}{4} \sum_{n=1}^{\infty}\left|x_{n}\right|^{2}, \quad \sum_{n=1}^{\infty} n^{2}\left|x_{n}+x_{n+1}\right|^{2} \geq \frac{1}{4} \sum_{n=1}^{\infty}\left|x_{n}\right|^{2}$.

Proof. If $\left\{y_{n}\right\} \in \ell^{1}$, then $y_{n} \rightarrow 0$, and $y_{n}=\sum_{i=n}^{\infty}\left(y_{i}-y_{i+1}\right)$. Therefore,

$$
\begin{aligned}
\sum_{n=1}^{\infty} y_{n} & =\sum_{n=1}^{\infty} \sum_{i=n}^{\infty}\left(y_{i}-y_{i+1}\right)=\sum_{i=1}^{\infty} \sum_{n=1}^{i}\left(y_{i}-y_{i+1}\right) \\
& =\sum_{i=1}^{\infty} i\left(y_{i}-y_{i+1}\right) \leq \sum_{i=1}^{\infty} i\left|y_{i}-y_{i+1}\right| .
\end{aligned}
$$

If $\left\{x_{n}\right\} \in \ell^{2}$ then $y_{n}=\left|x_{n}\right|^{2}$ defines a sequence in $\ell^{1}$ and the previous observation implies that

$$
\begin{aligned}
\sum_{n=1}^{\infty}\left|x_{n}\right|^{2} & \leq\left.\sum_{n=1}^{\infty}|| x_{n}\right|^{2}-\left|x_{n+1}\right|^{2} \mid \\
& =\sum_{n=1}^{\infty} n|| x_{n}|-| x_{n+1}|| \cdot|| x_{n}|+| x_{n+1}|| \\
& \leq\left(\sum_{n=1}^{\infty} n^{2}\left|x_{n}-x_{n+1}\right|^{2}\right)^{1 / 2} \cdot\left(\left.\sum_{n=1}^{\infty}|| x_{n}|+| x_{n+1}\right|^{2}\right)^{1 / 2} \\
& \leq\left(\sum_{n=1}^{\infty} n^{2}\left|x_{n}-x_{n+1}\right|^{2}\right)^{1 / 2} \cdot 2\left(\sum_{n=1}^{\infty}\left|x_{n}\right|^{2}\right)^{1 / 2} .
\end{aligned}
$$

Thus $\sum_{n=1}^{\infty} n^{2}\left|x_{n}-x_{n+1}\right|^{2} \geq \frac{1}{4} \sum_{n=1}^{\infty}\left|x_{n}\right|^{2}$. To obtain (ii), apply (i) to the sequence $\left\{(-1)^{n+1} x_{n}\right\}$.

TheOREM 1.3. If $a_{2 n}=a_{2 n-1}=n, n \geq 1, b_{n}=0, n \geq 1$, then $C$ defined in (1.1) has a spectral gap containing the origin.

Proof. Let $A^{+}$denote the restriction of $C^{2}$ to the subspace of $\ell^{2}$ spanned by $\left\{\phi_{2 k-1}\right\}_{k=1}^{\infty}$. Obtain $A^{-}$from $A^{+}$by negating each diagonal entry. Then

$$
A^{-}=\left[\begin{array}{ccccc}
-a_{2}^{2} & a_{2}^{2} & 0 & 0 & \cdots \\
a_{2}^{2} & -a_{2}^{2}-a_{4}^{2} & a_{4}^{2} & 0 & \cdots \\
0 & a_{4}^{2} & -a_{4}^{2}-a_{6}^{2} & a_{6}^{2} & \cdots \\
0 & 0 & a_{6}^{2} & -a_{6}^{2}-a_{8}^{2} & \cdots \\
. & . & . & . & .
\end{array}\right] .
$$

Since the row sums are identically zero, it follows from Theorem 2.2 in [DP95] (with $M=0$ ), that $\left\langle A^{-} x, x\right\rangle=-\sum_{n=1}^{\infty} n^{2}\left|x_{n+1}-x_{n}\right|^{2}$. By Lemma 1.2 above, $\sigma\left(A^{-}\right) \subset(-\infty,-1 / 4]$. If $W=\left[w_{i j}\right]$ is a diagonal operator 
with $w_{i i}=(-1)^{i+1}$, then $A^{+}=W\left(-A^{-}\right) W$, and it follows that $\sigma\left(A^{+}\right) \subset$ $[1 / 4, \infty)$. Hence $\sigma(C) \subset(-\infty,-1 / 2] \cup[1 / 2, \infty)$, which establishes the existence of a spectral gap.

Remark. It follows from [DP95, Theorem 2.3] applied to $A^{-}$with $M$ $=0$ that if $a_{2 n-1}=a_{2 n}$ for all $n$, and $a_{2 n} / n \rightarrow 0$, then 0 is in the spectrum of the operator defined in (1.1).

2. Two non-negative differences. If the diagonal sequence is identically zero, then the subdiagonal sequence $\left\{a_{n}\right\}, a_{n}>0$, completely determines the operator $C$ defined in (1.1). The following result characterizes the spectrum of a class of tridiagonal operators with zero diagonal in terms of a periodic difference sequence $\left\{d_{n}\right\}$ defined by $d_{n}=a_{n}-a_{n-1}$. (Note that in Theorem 1.1 above, $d_{2 n+1}=1, d_{2 n}=0$.)

Theorem 2.1. Assume $b_{n}=0, n \geq 1$. Fix $a>0, \delta \geq 0, d \geq 0$. Let

$$
\begin{aligned}
a_{2 n+1} & =a+n(\delta+d), & & n=0,1,2, \ldots, \\
a_{2 n} & =a+n(\delta+d)-d, & & n=1,2, \ldots
\end{aligned}
$$

Then $C$ defined in (1.1) is self-adjoint. If

$$
a-d+\frac{\delta}{2}+\frac{d}{2} \cdot \frac{\alpha^{2}}{a^{2}}>0
$$

then the corresponding spectral measure is absolutely continuous on $(-\infty,-\alpha) \cup(\alpha, \infty)$. In particular, if $a-d+\delta / 2>0$ then the spectral measure is absolutely continuous on $(-\infty, 0) \cup(0, \infty)$.

The proof of this theorem depends on the following result which was proved in [DP02].

Lemma 2.2. Let $C$ in (1.1) be a self-adjoint Jacobi matrix with a zero diagonal, generated by a positive sequence $\left\{a_{n}\right\}$. If $C=\int \lambda d E_{\lambda}$, and $\Delta$ is a Borel subset of $(0, \infty)$ then

$$
\sum_{n=1}^{\infty}\left|\left\langle E(\Delta) \phi_{1}, \phi_{2 n-1}\right\rangle\right|^{2}=\sum_{n=1}^{\infty}\left|\left\langle E(\Delta) \phi_{1}, \phi_{2 n}\right\rangle\right|^{2} .
$$

Proof of Theorem 2.1. The Carleman condition implies that $C$ is selfadjoint. A commutator equation will be used to study the corresponding spectral measure. Let $S$ be the unilateral shift defined on $\ell^{2}$ by $S \phi_{n}=\phi_{n+1}$, $n=1,2, \ldots$ Let $J=\left(S-S^{*}\right) / i$. Then $C J-J C=-2 i K$ where $K=\left[k_{i j}\right]$, and $k_{11}=a, k_{i i}=\delta$ if $i=2 n, k_{i i}=d$ if $i=2 n+1, k_{i, i+2}=k_{i+2, i}=\delta / 2$ if $i=2 n-1, k_{i, i+2}=k_{i+2, i}=d / 2$ if $i=2 n$, and $k_{i j}=0$ otherwise. That is, 


$$
K=\left[\begin{array}{ccccc}
a & 0 & \delta / 2 & 0 & \cdots \\
0 & \delta & 0 & d / 2 & \cdots \\
\delta / 2 & 0 & d & 0 & \ldots \\
0 & d / 2 & 0 & \delta & \ldots \\
\cdot & \cdot & \delta / 2 & . & \ldots
\end{array}\right] .
$$

Assume $C=\int \lambda d E_{\lambda}, \Delta$ is an interval which is a subset of $(\alpha, \infty), \alpha>0$, and $x=E(\Delta) \phi_{1}$. Then $x=\sum_{n=1}^{\infty} x_{n} \phi_{n}$ where $x_{n}=\left\langle E(\Delta) \phi_{1}, \phi_{n}\right\rangle$, and

$$
\begin{aligned}
\langle K x, x\rangle= & a\left|x_{1}\right|^{2}+d \sum_{k=1}^{\infty}\left|x_{2 k+1}\right|^{2}+d \sum_{k=1}^{\infty} x_{2 k} x_{2 k+2} \\
& +\delta \sum_{k=1}^{\infty}\left|x_{2 k}\right|^{2}+\delta \sum_{k=1}^{\infty} x_{2 k-1} x_{2 k+2} \\
\geq & a\left|x_{1}\right|^{2}+d \sum_{k=1}^{\infty}\left|x_{2 k+1}\right|^{2}-d \sum_{k=1}^{\infty}\left|x_{2 k}\right| \cdot\left|x_{2 k+2}\right| \\
& +\delta \sum_{k=1}^{\infty}\left|x_{2 k}\right|^{2}-\delta \sum_{k=1}^{\infty}\left|x_{2 k-1}\right| \cdot\left|x_{2 k+2}\right| \\
\geq & a\left|x_{1}\right|^{2}+d \sum_{k=1}^{\infty}\left|x_{2 k+1}\right|^{2}-\frac{d}{2} \sum_{k=1}^{\infty}\left[\left|x_{2 k}\right|^{2}+\left|x_{2 k+2}\right|^{2}\right] \\
& +\delta \sum_{k=1}^{\infty}\left|x_{2 k}\right|^{2}-\frac{\delta}{2} \sum_{k=1}^{\infty}\left[\left|x_{2 k-1}\right|^{2}+\left|x_{2 k+1}\right|^{2}\right] \\
\geq & a\left|x_{1}\right|^{2}+d \sum_{k=1}^{\infty}\left|x_{2 k+1}\right|^{2}-d \sum_{k=1}^{\infty}\left|x_{2 k}\right|^{2}+\frac{d}{2}\left|x_{2}\right|^{2} \\
& +\delta \sum_{k=1}^{\infty}\left|x_{2 k}\right|^{2}-\delta \sum_{k=1}^{\infty}\left|x_{2 k-1}\right|^{2}+\frac{\delta}{2}\left|x_{1}\right|^{2} .
\end{aligned}
$$

It now follows from Lemma 2.2 that

$$
\langle K x, x\rangle \geq(a-d)\left|x_{1}\right|^{2}+\frac{d}{2}\left|x_{2}\right|^{2}+\frac{\delta}{2}\left|x_{1}\right|^{2} .
$$

But

$$
x_{2}=\left\langle E(\Delta) \phi_{1}, E(\Delta) \phi_{2}\right\rangle=\int_{\Delta} P_{2}(x) d \mu>\frac{\alpha}{a} \mu(\Delta)=\frac{\alpha}{a}\left\langle E(\Delta) \phi_{1}, E(\Delta) \phi_{1}\right\rangle .
$$

Hence

$$
\langle K x, x\rangle>\left[a-d+\frac{\delta}{2}+\frac{d}{2}\left(\frac{\alpha}{a}\right)^{2}\right]\left|\left\langle E(\Delta) \phi_{1}, E(\Delta) \phi_{1}\right\rangle\right|^{2} .
$$

On the other hand, if $\lambda$ is the midpoint of the interval $\Delta$, then $C J-J C=$ 
$(C-\lambda I) J-J(C-\lambda I)$ and it follows that

$$
\begin{aligned}
\left|\left\langle K E(\Delta) \phi_{1}, E(\Delta) \phi_{1}\right\rangle\right| & \leq 2\|J\| \cdot\left\|E(\Delta)(C-\lambda I) \phi_{1}\right\| \\
& \leq\|J\| \cdot|\Delta| \cdot\left\|E(\Delta) \phi_{1}\right\|^{2}
\end{aligned}
$$

where $|\Delta|$ denotes the Lebesgue measure of the interval $\Delta$. Thus the two inequalities can be combined to show that if $\mu(\Delta)=\left\|E(\Delta) \phi_{1}\right\|^{2} \neq 0$ then

$$
\mu(\Delta) \leq\left(\frac{\|J\|}{a-d+\frac{\delta}{2}+\frac{d}{2}\left(\frac{\alpha}{a}\right)^{2}}\right)|\Delta|
$$

so that the measure is absolutely continuous.

Theorem 2.3. Assume that the sequences $\left\{a_{n}\right\},\left\{b_{n}\right\}$ are defined as in Theorem 2.1. Then $x=0$ is an eigenvalue for $C$ if and only if $\delta>d$.

Proof. It is well known that $x=0$ is an eigenvalue for $C$ if and only if $\sum_{n=1}^{\infty}\left|P_{n}(0)\right|^{2}<\infty$. Note that for $n=1,2, \ldots$,

$$
P_{2 n}(0)=0, \quad P_{2 n+1}(0)=(-1)^{n} \frac{a_{1} a_{2} \ldots a_{2 n-1}}{a_{2} a_{4} \ldots a_{2 n}} .
$$

Hence $\sum_{n=1}^{\infty}\left|P_{2 n+1}(0)\right|^{2}=\sum_{n=1}^{\infty} c_{n}$ and

$$
\frac{c_{n+1}}{c_{n}}=\left(\frac{a_{2 n+1}}{a_{2 n+2}}\right)^{2}=\left(\frac{a+n(d+\delta)}{a+\delta+n(d+\delta)}\right)^{2}=\frac{n^{2}+\frac{2 a}{d+\delta} n+\left(\frac{a}{d+\delta}\right)^{2}}{n^{2}+\frac{2(a+\delta)}{d+\delta} n+\left(\frac{a+\delta}{d+\delta}\right)^{2}} .
$$

By Gauss' Test [Kno47, p. 284], $\sum_{n=1}^{\infty} c_{n}$ converges if and only if

$$
\frac{2 a}{d+\delta}-\frac{2(a+\delta)}{d+\delta}<-1
$$

Hence $x=0$ is an eigenvalue for $C$ if and only if $2 \delta /(d+\delta)>1 \Leftrightarrow \delta>d$.

Note that Theorem 1.1 follows from Theorems 2.1 and 2.3 when $d=$ $a=1$ and $\delta=0$. It is also interesting to observe that by Theorem 2.1, a sufficiently large choice of the parameter $a$ will imply absolute continuity on $(-\infty, 0) \cup(0, \infty)$ for any given values of $d$ and $\delta$.

Theorem 2.4. Assume $a_{2 n-1}=a_{2 n}=n^{\alpha}, 0<\alpha<1, b_{n}=0$. Then the operator $C$ defined in (1.1) has no eigenvalues. The spectrum of $C$ is the real line.

Proof. Define $\alpha_{2 n-1}=\alpha_{2 n}=n^{1-\alpha}, n=1,2, \ldots$ Let

$$
J=-i\left[\begin{array}{ccccc}
0 & -\alpha_{1} & 0 & 0 & \ldots \\
\alpha_{1} & 0 & -\alpha_{2} & 0 & \ldots \\
0 & \alpha_{2} & 0 & -\alpha_{3} & \ldots \\
0 & 0 & \alpha_{3} & 0 & \ldots \\
. & . & . & . & \ldots
\end{array}\right]
$$


If $C J-J C=-2 i K$, then it will be shown below that $K=\left[k_{i j}\right]$ is bounded. Matrix multiplication shows that the non-zero entries of $K$ are given by

$$
\begin{aligned}
2 k_{2 n, 2 n+2} & =2 k_{2 n+2,2 n}=(n+1)^{\alpha} n^{1-\alpha}-n^{\alpha}(n+1)^{1-\alpha}, \\
k_{2 n+1,2 n+1} & =a_{2 n+1} \alpha_{2 n+1}-a_{2 n} \alpha_{2 n}=1 .
\end{aligned}
$$

To estimate the non-zero off-diagonal entries of $K$, let

$$
\begin{aligned}
f(n) & =2 k_{2 n+2,2 n}=\frac{(n+1)^{\alpha}}{n^{\alpha}} n-\frac{n^{\alpha}}{(n+1)^{\alpha}}(n+1) \\
& =\frac{(n+1)^{2 \alpha} n-n^{2 \alpha}(n+1)}{n^{\alpha}(n+1)^{\alpha}}=\frac{\left(1+\frac{1}{n}\right)^{2 \alpha}-\left(1+\frac{1}{n}\right)}{\frac{1}{n}} \cdot \frac{1}{\left(1+\frac{1}{n}\right)^{\alpha}} .
\end{aligned}
$$

So $f(1)=2^{\alpha}-2^{1-\alpha}$ and if $g(x)=x^{2 \alpha}-x$ then $\lim _{n \rightarrow \infty} f(n)=g^{\prime}(1)=$ $2 \alpha-1$. Observe that

(i) if $0<\alpha<1 / 2$ then $f(n)$ decreases to $-1+2 \alpha$ and $-1<f(1)<0$,

(ii) if $\alpha=1 / 2$ then $f(n)=0$ for all $n$,

(iii) if $1 / 2<\alpha<1$ then $f(n)$ increases to $-1+2 \alpha$ and $0<f(1)<1$.

Now assume that $\nu \neq 0$ is an eigenvalue for $C$. If $C x=\nu x$ then $x=\left\{P_{n}(\nu)\right\}, \sum_{n=1}^{\infty}\left|P_{n}(\nu)\right|^{2}<\infty$. It follows from (1.2) and the fact that $b_{n}=0$ for all $n$ that $P_{2 n-1}(-x)=P_{2 n-1}(x), P_{2 n}(-x)=-P_{2 n}(x)$. Hence $\nu$ is an eigenvalue if and only if $-\nu$ is an eigenvalue. Since eigenvectors corresponding to distinct eigenvalues of self-adjoint operators are orthogonal,

$$
0=\sum_{n=1}^{\infty}\left\langle P_{n}(\nu), P_{n}(-\nu)\right\rangle=\sum_{n=1}^{\infty}\left|P_{2 n-1}(\nu)\right|^{2}-\sum_{n=1}^{\infty}\left|P_{2 n}(\nu)\right|^{2} .
$$

Therefore

$$
\begin{aligned}
\langle K x, x\rangle & =\sum_{n=1}^{\infty}\left|P_{2 n-1}(\nu)\right|^{2}+\sum_{n=1}^{\infty} f(n) P_{2 n}(\nu) P_{2 n+2}(\nu) \\
& \geq \sum_{n=1}^{\infty}\left|P_{2 n-1}(\nu)\right|^{2}-\sum_{n=1}^{\infty}\left|P_{2 n}(\nu)\right| \cdot\left|P_{2 n+2}(\nu)\right| \\
& \geq \sum_{n=1}^{\infty}\left|P_{2 n-1}(\nu)\right|^{2}+\frac{1}{2}\left|P_{2}(\nu)\right|^{2}-\sum_{n=1}^{\infty}\left|P_{2 n}(\nu)\right|^{2} \\
& \geq \frac{1}{2}\left|P_{2}(\nu)\right|^{2} .
\end{aligned}
$$

On the other hand, if $x=\left\{P_{n}(\nu)\right\}$ and $x_{N}=\left\{P_{n}(\nu)\right\}_{n=1}^{N}$, then it follows from the equation $(C-\nu I) J-J(C-\nu I)=-2 i K$ that $\left|\left\langle J C_{\nu} x_{N}, x\right\rangle\right|=$ $2\left|\left\langle K x_{N}, x\right\rangle\right|$ where $C_{\nu}=C-\nu I$. Since $K$ is bounded,

$$
\lim _{N \rightarrow \infty}\left|\left\langle J C_{\nu} x_{N}, x\right\rangle\right|=\lim _{N \rightarrow \infty}\left|2\left\langle K x_{N}, x\right\rangle\right|=2|\langle K x, x\rangle| .
$$


But the following argument will show that $\lim _{N \rightarrow \infty}\left|\left\langle J C_{\nu} x_{N}, x\right\rangle\right|=0$. For assume that $\lim _{N \rightarrow \infty}\left|\left\langle J C_{\nu} x_{N}, x\right\rangle\right|=p, p>0$. A direct computation shows that

$\left|\left\langle J C_{\nu} x_{N}, x\right\rangle\right|$

$=\left|\alpha_{N-1} a_{N} P_{N+1}(\nu) P_{N-1}(\nu)-\alpha_{N} a_{N} P_{N}(\nu) P_{N}(\nu)-\alpha_{N} a_{N} P_{N+1}(\nu) P_{N+1}(\nu)\right|$.

For large $N=2 k$, the inequality

$$
\frac{p}{2}<\left|\left\langle J C_{\nu} x_{N}, x\right\rangle\right| \leq k\left[\left|P_{N+1}(\nu) P_{N-1}(\nu)\right|+\left|P_{N}(\nu)\right|^{2}+\left|P_{N+1}(\nu)\right|^{2}\right]
$$

contradicts the fact that

$$
\sum_{k=1}^{\infty}\left[\left|P_{2 k+1}(\nu) P_{2 k-1}(\nu)\right|+\left|P_{2 k}(\nu)\right|^{2}+\left|P_{2 k+1}\right|^{2}\right]<\infty .
$$

Thus $\langle K x, x\rangle=0$, which contradicts (2.1). Hence there are no non-zero eigenvalues. A direct computation (see the proof of Theorem 2.3) shows that 0 is also not an eigenvalue.

It now remains to locate the spectrum of $C$. To do that, define $H$ by (1.1) with $a_{n}=n^{\alpha}, b_{n}=0$. Then $a_{2 n}=(2 n)^{\alpha}, a_{2 n-1}=(2 n-1)^{\alpha}$. It will be shown below that $2^{\alpha} C-H$ is compact. To see this note that the non-zero entries of $2^{\alpha} C-H$ are

$$
2^{\alpha} n^{\alpha}-(2 n-1)^{\alpha}=2^{\alpha}\left(n^{\alpha}-(n-1 / 2)^{\alpha}\right)=(2 n)^{\alpha-1} \frac{1-\left(1-\frac{1}{2 n}\right)^{\alpha}}{\frac{1}{2 n}} .
$$

Let $f(x)=x^{\alpha}$. Then

$$
\frac{1-\left(1-\frac{1}{2 n}\right)^{\alpha}}{\frac{1}{2 n}} \rightarrow f^{\prime}(1)
$$

Thus $2^{\alpha} n^{\alpha}-(2 n-1)^{\alpha} \rightarrow 0$. It follows that the two sets $\sigma(C), \sigma(H)$ have the same set of limit points. Since it is known that $\sigma(H)$ is the real line (see [JN99a] and [JN99b]), the same conclusion holds for $C$ as claimed.

3. Spectral transition parameters. In this section we investigate the spectrum of the operator in (1.1) as a function of a parameter which will be introduced in the first result.

TheOREM 3.1. Assume that $b_{n}=0, a_{2 n-1}=n^{\alpha}, a_{2 n}=\delta n^{\alpha}, n=$ $1,2, \ldots$, with $\delta>0,0<\alpha \leq 1$. Then $C$ defined in (1.1) is self-adjoint. If $\delta=1$, then there are no eigenvalues. If $\delta \neq 1$, then $\sigma(C)$ is discrete with no accumulation points.

Proof. Let $A^{+}$denote the restriction of $C^{2}$ to the subspace of $\ell^{2}$ spanned by $\left\{\phi_{2 k-1}\right\}_{k=1}^{\infty}$. Then 


$$
\begin{aligned}
\lim _{n \rightarrow \infty} \frac{\left[a_{2 n-2}^{2}+a_{2 n-1}^{2}\right]^{2}}{a_{2 n-3}^{2} a_{2 n-2}^{2}-a_{2 n-1}^{2} a_{2 n}^{2}} & =\lim _{n \rightarrow \infty} \frac{\left[\delta^{2}(n-1)^{2 \alpha}+n^{2 \alpha}\right]^{2}}{\delta^{2}(n-1)^{2 \alpha}+\delta^{2} n^{2 \alpha} n^{2 \alpha}} \\
& =\frac{\delta^{4}+2 \delta^{2}+1}{2 \delta^{2}} .
\end{aligned}
$$

If $\delta \neq 1$ then

$$
\frac{\delta^{4}+2 \delta^{2}+1}{2 \delta^{2}}>2
$$

and it follows from [JN99a, Theorem 4.1] that $A^{+}$, and hence $C$ by [DP02], has a pure point spectrum.

4. Spectral gaps. It was shown in Theorem 1.3 that if $C$ is defined by (1.1) with $a_{2 n}=a_{2 n-1}=n, n \geq 1, b_{n}=0, n \geq 1$, then $(-1 / 2,1 / 2)$ is a gap in the spectrum of $C$. This section will consider a period two perturbation of this result. Specifically it will be assumed that

$$
a_{2 n-1}=n+c \quad \text { and } \quad a_{2 n}=n+d, \quad n=1,2, \ldots
$$

It will also be assumed that $c>-1$ so that $a_{1}>0$, and that $0<d-c<1$ so that the sequence $\left\{a_{k}\right\}$ is monotone. Further technical conditions will be imposed on the parameters $c$ and $d$ to establish the existence of a spectral gap.

THEOREM 4.1. Assume that $a_{2 n-1}=n+c, a_{2 n}=n+d$, for $n=1,2, \ldots$, with fixed parameters $c$ and $d$ chosen so that $c>-1, c+d>0$. Let

$$
\alpha=(d-c)^{2}-(d-c)+1 / 4 .
$$

Then $C$ defined by (1.1) is self-adjoint, and if $\alpha>0$, then $(-\sqrt{\alpha}, \sqrt{\alpha})$ is a gap in the essential spectrum of $C$.

Proof. Since $\sum_{n=1}^{\infty} 1 / a_{n}=\infty$, the operator $C$ defined in (1.1) is selfadjoint. In the argument that follows it will initially be assumed that all sequences $x=\left\{x_{n}\right\}$ have a finite number of non-zero entries. Let $A^{+}$denote the restriction of $C^{2}$ to the subspace of $\ell^{2}$ spanned by $\left\{\phi_{2 k-1}\right\}_{k=1}^{\infty}$. Since

$$
A^{+}=\left[\begin{array}{ccccc}
a_{1}^{2} & a_{1} a_{2} & 0 & 0 & \cdots \\
a_{1} a_{2} & a_{2}^{2}+a_{3}^{2} & a_{3} a_{4} & 0 & \cdots \\
0 & a_{3} a_{4} & a_{4}^{2}+a_{5}^{2} & a_{5} a_{6} & \ldots \\
0 & 0 & a_{5} a_{6} & a_{6}^{2}+a_{7}^{2} & \ldots \\
. & . & . & . & \ldots
\end{array}\right] .
$$

we have

$$
\begin{aligned}
& \left\langle A^{+} x, x\right\rangle=a_{1}^{2}\left|x_{1}\right|^{2}+a_{1} a_{2} x_{2} \bar{x}_{1} \\
& +\sum_{k=2}^{\infty}\left[a_{2 k-2} a_{2 k-3} x_{k-1} \bar{x}_{k}+\left(a_{2 k-2}^{2}+a_{2 k-1}^{2}\right)\left|x_{k}\right|^{2}+a_{2 k-1} a_{2 k} x_{k+1} \bar{x}_{k}\right]
\end{aligned}
$$




$$
\begin{aligned}
& =a_{1}^{2}\left|x_{1}\right|^{2}+a_{1} a_{2} x_{2} \bar{x}_{1} \\
& +\sum_{k=2}^{\infty}\left(a_{2 k-2} a_{2 k-3}+a_{2 k-2}^{2}+a_{2 k-1}^{2}+a_{2 k-1} a_{2 k}\right)\left|x_{k}\right|^{2} \\
& +\sum_{k=2}^{\infty}\left[a_{2 k-2} a_{2 k-3}\left(x_{k-1}-x_{k}\right) \bar{x}_{k}+a_{2 k-1} a_{2 k}\left(x_{k+1}-x_{k}\right) \bar{x}_{k}\right] \\
& =\left(a_{1}^{2}+a_{1} a_{2}\right)\left|x_{1}\right|^{2}+\sum_{k=2}^{\infty}\left(a_{2 k-2} a_{2 k-3}+a_{2 k-2}^{2}+a_{2 k-1}^{2}+a_{2 k-1} a_{2 k}\right)\left|x_{k}\right|^{2} \\
& -\sum_{k=1}^{\infty} a_{2 k-1} a_{2 k}\left|x_{k+1}-x_{k}\right|^{2} \\
& =\left(-a_{1}^{2}+a_{1} a_{2}\right)\left|x_{1}\right|^{2}+\sum_{k=2}^{\infty}\left(a_{2 k-2} a_{2 k-3}-a_{2 k-2}^{2}-a_{2 k-1}^{2}+a_{2 k-1} a_{2 k}\right)\left|x_{k}\right|^{2} \\
& -\sum_{k=1}^{\infty} a_{2 k-1} a_{2 k}\left|x_{k+1}-x_{k}\right|^{2}+2 a_{1}^{2}\left|x_{1}\right|^{2}+2 \sum_{k=2}^{\infty}\left[a_{2 k-2}^{2}+a_{2 k-1}^{2}\right]\left|x_{k}\right|^{2} \\
& =\left((d-c)(1+c)\left|x_{1}\right|^{2}+(d-c)(1+c-d) \sum_{k=2}^{\infty}\left|x_{k}\right|^{2}\right) \\
& +\left(2 a_{1}^{2}\left|x_{1}\right|^{2}+2 \sum_{k=2}^{\infty}\left[a_{2 k-2}^{2}+a_{2 k-1}^{2}\right]\left|x_{k}\right|^{2}-\sum_{k=1}^{\infty} a_{2 k-1} a_{2 k}\left|x_{k+1}-x_{k}\right|^{2}\right) \\
& =S_{1}+S_{2} .
\end{aligned}
$$

Clearly,

$$
S_{1}=d(d-c)\left|x_{1}\right|^{2}+(d-c)(1+c-d) \sum_{k=1}^{\infty}\left|x_{k}\right|^{2} .
$$

On the other hand,

$$
\begin{aligned}
S_{2}= & 2(1+c)^{2}\left|x_{1}\right|^{2}+2 \sum_{k=2}^{\infty}\left[(k-1+d)^{2}+(k+c)^{2}\right]\left|x_{k}\right|^{2} \\
& -\sum_{k=1}^{\infty}(k+c)(k+d)\left|x_{k+1}-x_{k}\right|^{2} \\
= & 2\left|x_{1}\right|^{2}+2 \sum_{k=2}^{\infty}\left[(k-1)^{2}+k^{2}\right]\left|x_{k}\right|^{2}-\sum_{k=1}^{\infty} k^{2}\left|x_{k+1}-x_{k}\right|^{2} \\
& +2\left(2 c+c^{2}\right)\left|x_{1}\right|^{2}+2 \sum_{k=2}^{\infty}\left[2 d(k-1)+d^{2}+2 c k+c^{2}\right]\left|x_{k}\right|^{2} \\
& -\sum_{k=1}^{\infty}(c k+d k+c d)\left|x_{k+1}-x_{k}\right|^{2}
\end{aligned}
$$




$$
\begin{aligned}
= & \sum_{k=1}^{\infty} k^{2}\left|x_{k+1}+x_{k}\right|^{2}+2\left(2 c+c^{2}\right)\left|x_{1}\right|^{2} \\
& +2 \sum_{k=2}^{\infty}\left[2 d(k-1)+d^{2}+2 c k+c^{2}\right]\left|x_{k}\right|^{2} \\
& -\sum_{k=1}^{\infty}(c k+d k+c d)\left|x_{k+1}-x_{k}\right|^{2} .
\end{aligned}
$$

It follows from Lemma 1.2 that

$$
\begin{aligned}
S_{2} \geq & \sum_{k=1}^{\infty} \frac{1}{4}\left|x_{k}\right|^{2}+2\left(2 c+c^{2}\right)\left|x_{1}\right|^{2}+2 \sum_{k=2}^{\infty}\left[2 d(k-1)+d^{2}+2 c k+c^{2}\right]\left|x_{k}\right|^{2} \\
& -\sum_{k=1}^{\infty}(c k+d k+c d)\left|x_{k+1}-x_{k}\right|^{2} .
\end{aligned}
$$

Since it was assumed that $c+d>0$ it follows that for some $k_{0} \geq 1, k_{0}(c+d)$ $\geq c d$. Thus

$$
\begin{aligned}
\left\langle A^{+} x, x\right\rangle \geq & d(d-c)\left|x_{1}\right|^{2}+(d-c)(1+c-d) \sum_{k=1}^{\infty}\left|x_{k}\right|^{2}+\sum_{k=1}^{\infty} \frac{1}{4}\left|x_{k}\right|^{2} \\
& +2\left(2 c+c^{2}\right)\left|x_{1}\right|^{2}+2 \sum_{k=2}^{\infty}\left[2 d(k-1)+d^{2}+2 c k+c^{2}\right]\left|x_{k}\right|^{2} \\
& -2 \sum_{k=k_{0}}^{\infty}(c k+d k+c d)\left[\left|x_{k+1}\right|^{2}+\left|x_{k}\right|^{2}\right] .
\end{aligned}
$$

Let

$$
\alpha=(d-c)^{2}-(d-c)+1 / 4 .
$$

Then $\left\langle A^{+} x, x\right\rangle \geq \alpha\langle x, x\rangle$ for all $x$ in the domain of $A^{+}$, orthogonal to $\left\{\phi_{1}, \ldots, \phi_{k_{0}}\right\}$. By [RS78, Theorem XIII.1], $(-\sqrt{\alpha}, \sqrt{\alpha})$ is a gap in the essential spectrum of $C$.

The proof of the following result is almost identical to the proof of Theorem 4.1. The assumptions, however, are mutually exclusive.

TheOREM 4.2. Assume that $b_{n}=0, a_{2 n-1}=n-d, a_{2 n}=n+d, n=$ $1,2, \ldots$, with $0<d<1 / 8$. Then $C$ defined by (1.1) is self-adjoint, and $(-\sqrt{1 / 4-2 d}, \sqrt{1 / 4-2 d})$ is a gap in the spectrum of $C$.

Proof. The Carleman condition $\sum_{n=1}^{\infty} 1 / a_{n}=\infty$ implies that $C$ is selfadjoint. The argument presented in the proof Theorem 4.1 can be used to establish the following inequality: 


$$
\begin{aligned}
\left\langle A^{+} x, x\right\rangle \geq & d(d-c)\left|x_{1}\right|^{2}+(d-c)(1+c-d) \sum_{k=1}^{\infty}\left|x_{k}\right|^{2}+\sum_{k=1}^{\infty} \frac{1}{4}\left|x_{k}\right|^{2} \\
& +2\left(2 c+c^{2}\right)\left|x_{1}\right|^{2}+2 \sum_{k=2}^{\infty}\left[2 d(k-1)+d^{2}+2 c k+c^{2}\right]\left|x_{k}\right|^{2} \\
& -\sum_{k=1}^{\infty}(c k+d k+c d)\left|x_{k+1}\right| x_{k+1}-\left.x_{k}\right|^{2}
\end{aligned}
$$

Since $c=-d$, and hence $c+d=0, c d \leq 0$, it follows that

$$
\left\langle A^{+} x, x\right\rangle \geq(-2 d+1 / 4) \sum_{k=1}^{\infty}\left|x_{k}\right|^{2} \text {. }
$$

Thus if $\alpha=-2 d+1 / 4, \alpha>0$, it follows that $\left\langle A^{+} x, x\right\rangle \geq \alpha\langle x, x\rangle$ for all $x$ in the domain of $A^{+}$, and hence that $(-\sqrt{\alpha}, \sqrt{\alpha})$ is a gap in the spectrum of $C$.

Theorems 2.1 and 4.1 can be used to prove the following result which extends both Theorem 1.1 and Theorem 1.3.

THEOREM 4.3. If $b_{n}=0, a_{2 n-1}=n, a_{2 n}=n+d, n=1,2, \ldots$, and $0 \leq$ $d<1 / 2$, then the operator $C$ defined by (1.1) is self-adjoint and absolutely continuous, and $(-1 / 2+d, 1 / 2-d)$ is a gap in the spectrum.

THEOREM 4.4. Assume $a_{2 n-1}=n+c, a_{2 n}=n+d$, and let $C$ be the operator determined by (1.1). If $-1<c, d+c>0,0 \leq d-c \leq 1$ and $\alpha=(d-c)^{2}-(d-c)+1 / 4$, then for $\alpha>0,(-\sqrt{\alpha}, \sqrt{\alpha})$ is a gap in the essential spectrum of $C$. There are no non-zero eigenvalues in this gap, and the spectral measure $\mu$ is absolutely continuous on the complement of this interval.

Acknowledgements. The authors would like to thank Qingbo Huang for the proof of Lemma 1.2. Also, the problems considered in this paper were motivated in part by questions posed by G. Stolz, S. Naboko and J. Janas at a research seminar at the Banach Center in Warsaw.

\section{References}

[DP95] J. Dombrowski and S. Pedersen, Orthogonal polynomials, spectral measures and absolute continuity, J. Comput. Appl. Math. 65 (1995), 115-124.

[DP02] - - - Absolute continuity for Jacobi matrices with constant row sums, J. Math. Anal. Appl. 267 (2002), 695-713.

[JN99a] J. Janas and S. Naboko, Multithreshold spectral phase transition examples in a class of unbounded Jacobi matrices, Research Reports in Math. 7, 1999, Dept. Math., Stockholm Univ., Sweden. 
[JN99b] J. Janas and S. Naboko, Jacobi matrices with power-like weights-grouping in blocks approach, J. Funct. Anal. 166 (1999), 218-243.

[Kno47] K. Knopp, Theory and Application of Infinite Series, 2nd ed., Blackie, London and Glasgow, 1947.

[RS78] M. Reed and B. Simon, Methods of Modern Mathematical Physics IV: Analysis of Operators, Academic Press, New York, 1978.

Department of Mathematics and Statistics

Wright State University

Dayton, OH 45435, U.S.A.

E-mail: joanne.dombrowski@wright.edu steen.pedersen@wright.edu

Received February 27, 2001

Revised version March 20, 2001 